\title{
Performance Analysis on Using Period-One Oscillation of Optically Injected Semiconductor Lasers for Radio-over-Fiber Uplinks
}

\author{
Cuicui Cui and Sze-Chun Chan, Member, IEEE
}

\begin{abstract}
Nonlinear period-one (P1) dynamics of a semiconductor laser are investigated for radio-over-fiber uplink transmission. By optical injection locking, the laser in a base station is driven into the P1 oscillation state, which is further locked by the uplink microwave signal through modulation on the bias current. Due to double locking by both the optical injection and current modulation, the uplink microwave signal is converted into an optical signal for transmission to the central office. Comprehensive numerical simulations reveal that the proposed uplink transmission based on the P1 state provides wide, continuous, and optically-controlled tunability for the uplink subcarrier frequency, which exceeds the laser modulation bandwidth. The laser with a relaxation resonance frequency of only $10.25 \mathrm{GHz}$ is shown to support subcarrier frequencies reaching $60 \mathrm{GHz}$. Compared to the commonly used stable injection locking state and the free-running state, the proposed P1 state generates the microwave oscillation by the inherent nonlinear dynamics and thus reduces the requirement on the uplink signal strength for low-error transmission. Both electrical demodulation and all-optical demodulation are investigated, where the latter is found to be better in terms of the immunity to dispersion and the speed requirement on optoelectronic conversion. The results illustrate the capability of using the P1 oscillation state for optically controlled uplink transmissions.
\end{abstract}

Index Terms-Injection-locked oscillators, nonlinear dynamics, optical injection, radio-over-fiber, semiconductor lasers.

\section{INTRODUCTION}

$\mathbf{N}$ ONLINEAR dynamics of semiconductor lasers have been investigated for a variety of photonic microwave applications over the past few years. Even a single-mode laser under proper perturbation can exhibit a variety of nonlinear dynamics. Due to the short photon and electron lifetimes in semiconductor lasers, microwave waveforms can be obtained from photodetection of the laser emissions under nonlinear dynamics. Some recent applications include injection locking dynamics for signal inversion [1]-[3], periodic oscillation dynamics for microwave generation [4], [5], and chaotic dynamics for communication, ranging, and random bit

Manuscript received September 4, 2011; revised December 27, 2011; accepted January 17, 2012. Date of publication January 23, 2012; date of current version February 14, 2012. This work was supported in part by a grant from the City University of Hong Kong under Project 7002674 and a grant from the Research Grant Council of Hong Kong, China under Project CityU 111308.

The authors are with the Department of Electronic Engineering, City University of Hong Kong, Hong Kong, China (e-mail: tracy.cuicuicui@gmail.com; scchan@cityu.edu.hk).

Color versions of one or more of the figures in this paper are available online at http://ieeexplore.ieee.org.

Digital Object Identifier 10.1109/JQE.2012.2185487 generation [6]-[9]. In order to study these nonlinear dynamics, a number of perturbation schemes such as external continuouswave injection [10]-[14], pulsed injection [15], modulated injection [16], [17], mutual injection [18], optoelectronic feedback [19], optical feedback [20], [21], and combined perturbation [22] have been considered.

Among the different schemes, external optical injection scheme has attracted most attention for radio-over-fiber (RoF) communication [23]-[28]. With the advance of wireless communication systems, RoF has emerged as a promising technology for the next generation wireless communication applications. The approach employs an optical carrier modulated by a subcarrier microwave signal, which is then transmitted between the central office and remote base stations through optical fibers. RoF has the attractive advantages of centralizing high-speed electronics, low signal attenuation, large bandwidth, immunity to radio frequency interference, and high cell density [29]-[34]. By operating the injected laser in the stable locking state, RoF downlinks were reported [35]-[37]. Optical injection was applied for enhancing the bandwidth of the laser to allow direct modulation of the subcarrier microwave signal [24], [25], [35], [38]. However, the stable locking state still requires a relatively strong current modulation and optical injection. As a result, the period-one (P1) oscillation state was demonstrated recently for the uplink [39]. The subcarrier modulation was inherently generated by the P1 dynamics without requiring current modulation [40]. Using simply a 2.5-Gbps-grade single-mode semiconductor laser, RoF uplink transmission was realized at $16 \mathrm{GHz}$ with biterror rate (BER) remaining below $10^{-9}$ over a temperature range of at least $10{ }^{\circ} \mathrm{C}$ [39]. The approach was demonstrated without using specially designed multisection lasers [41] or external optical modulators required in various wavelength reuse techniques [42], [43]. Only ordinary single-mode lasers were employed in the proposed implementation [39].

In this paper, the performance of using the P1 oscillation state of an optically injected semiconductor laser for RoF uplink transmission is systematically investigated. Due to the induced instability in the coupling between the gain medium and the circulating optical field, the laser in the P1 state behaves as a photonic microwave oscillator. The oscillation frequency can be tuned continuously far beyond the relaxation resonance frequency. When a weak microwave uplink signal near the P1 oscillation frequency is applied to the injected laser, it is double-locked by the optical injection and the uplink signal. Through detailed simulation, it is found that the P1 state 
offers the following unique advantages. First, the generated microwave modulation is stronger and contains less phase noise for the P1 state as compared to the stable locking state. Second, as the uplink signal power increases, the BER reduces more quickly for the P1 state than for the stable locking state. Lastly, the optical spectrum is asymmetric to the central optical frequency, which leads to little chromatic dispersioninduced power penalty for electrical demodulation. No highspeed electronics is required for demodulation if all-optical demodulation is adopted. Additionally, unlike conventional direct modulation on lasers without optical injection, the approach using the P1 state is not limited by the modulation bandwidth and thus supports uplink frequencies beyond the relaxation frequency of the laser.

The results of the paper illustrate in detail the performances of using the P1 state for uplink at a range of frequencies, locking qualities, data rates, demodulation schemes, dispersion, and noise through comprehensive numerical simulation; although preliminary results at one particular uplink frequency using an optical demodulation scheme was demonstrated experimentally [39]. The numerical model is based on the rate equations, which were employed in analyzing dependencies on the microwave power [44]. Following this introduction, the RoF uplink system setup is introduced in Section II. The simulation model is presented in Section III. Then, based on the simulation model, detailed numerical results on the system performance with respect to the injection condition, uplink signal, and chromatic dispersion are addressed in Section IV. They are followed by a conclusion in Section V.

\section{SETUP}

Figure 1 shows the schematic of the proposed RoF uplink setup. A master laser at the central office optically injects into a single-mode semiconductor slave laser at the base station through a circulator and a single-mode fiber. The master laser gives continuous-wave emission detuned at frequency $f_{\mathrm{i}}$ above the free-running frequency of the slave laser. The downstream inset in Fig. 1 shows the optical spectrum that is offset by the free-running frequency. The detuning frequency $f_{\mathrm{i}}$ and the injection strength $\xi_{\mathrm{i}}$ can be controlled by the bias current and temperature of the master laser. By operating the system above the Hopf bifurcation line in the parameter space $\left(\xi_{\mathrm{i}}, f_{\mathrm{i}}\right)$, the slave laser experiences undamping of relaxation oscillation and thus exhibits P1 oscillation at a microwave frequency $f_{0}$ [26]. The upstream inset of Fig. 1 shows the slave laser emission spectrum, offset to the free-running frequency, that comprises of a regenerated component at $f_{\mathrm{i}}$ and a $\mathrm{P} 1$ component at $f_{\mathrm{i}}-f_{0}$. The generated $\mathrm{P} 1$ frequency $f_{0}$ can be widely tuned by varying $\left(\xi_{\mathrm{i}}, f_{\mathrm{i}}\right)$.

The uplink electrical signal at subcarrier frequency $f_{\mathrm{m}}$ is directly modulated onto the bias current of the slave laser. The signal strength is specified by the current modulation index $m$ on the bias current. Through controlling the optical injection parameters $\left(\xi_{\mathrm{i}}, f_{\mathrm{i}}\right)$, the frequency $f_{0}$ can be varied to the desired uplink subcarrier frequency $f_{\mathrm{m}}$, thereby locking the $\mathrm{P} 1$ oscillation. The slave laser is said to be double-locked by both

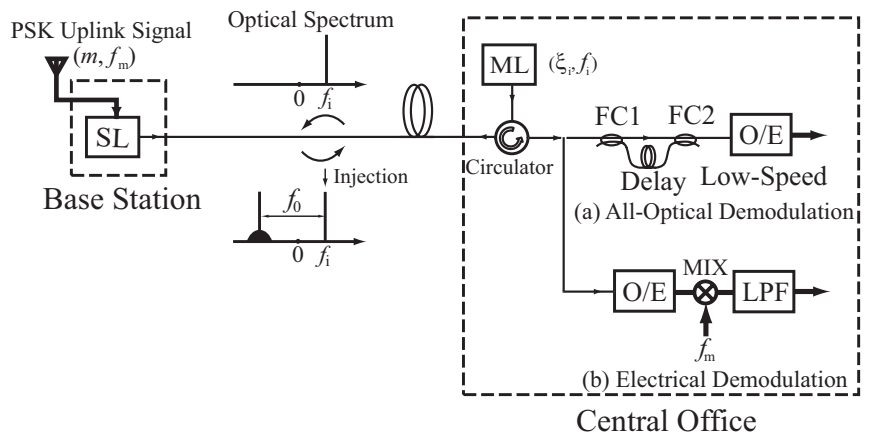

Fig. 1. Schematic of the RoF uplink using an optically injected laser. SL: slave laser. ML: master laser. LPF: low pass filter. MIX: microwave mixer. FC: 3-dB fiber coupler. Thin and thick lines correspond to optical and microwave paths, respectively.

the optical injection and the uplink current modulation [40]. Suppose the uplink signal now carries data with binary phaseshift keying (PSK). The phase of the locked P1 oscillation is encoded with the data accordingly. Thus, the optical sideband at $f_{\mathrm{i}}-f_{0}$ carries optical PSK data, which is transmitted back to the central office for demodulation.

Figures 1(a) and (b) illustrate two demodulation schemes in the central office. Figure 1(a) shows an all-optical demodulation scheme. The optical signal is first sent to an optical fiber delay-line interferometer, which consists of two 3 -dB fiber couplers and a fiber of one-bit delay. Consecutive bits are therefore interfered optically in realizing differential demodulation upon detection by an optical-to-electrical (O/E) converter. Such delayed homodyne technique requires only a low-speed O/E converter at the baseband [39]. Figure 1(b) presents an electrical demodulation scheme. The optical signal that carries the PSK data is first beat down to a microwave signal at a high-speed $\mathrm{O} / \mathrm{E}$ converter. The electrical microwave signal is then further down-converted by a local microwave oscillator using a microwave mixer, which is followed by a low-pass filter to obtain the baseband data. For comparison throughout the simulations, the cutoff frequencies of both the low-speed O/E in Fig. 1(a) and the low-pass filter in Fig. 1(b) are kept at four times the data rate. The performance of demodulation is monitored by the recovered eye-diagram in the investigation of the RoF uplink.

\section{Simulation Model}

The slave laser is a single-mode semiconductor laser that is adequately modeled as a Class B laser because the relaxation time of the material polarization is much shorter than the electron and photon lifetimes [1], [26], [45]-[47]. The dynamics of the slave laser are described by the normalized intracavity optical field amplitude $a_{\mathrm{r}}+\mathrm{i} a_{\mathrm{i}}$ and the normalized charge carrier density $\tilde{n}$ that are governed by the following rate equations [26]:

$$
\begin{aligned}
\frac{\mathrm{d} a_{\mathrm{r}}}{\mathrm{d} t}= & \frac{1}{2}\left[\frac{\gamma_{\mathrm{c}} \gamma_{\mathrm{n}}}{\gamma_{\mathrm{s}} \tilde{J}} \tilde{n}-\gamma_{\mathrm{p}}\left(a_{\mathrm{r}}^{2}+a_{\mathrm{i}}^{2}-1\right)\right]\left(a_{\mathrm{r}}+b a_{\mathrm{i}}\right) \\
& +\xi_{\mathrm{i}} \gamma_{\mathrm{c}} \cos 2 \pi f_{\mathrm{i}} t, \\
\frac{\mathrm{d} a_{\mathrm{i}}}{\mathrm{d} t}= & \frac{1}{2}\left[\frac{\gamma_{\mathrm{c}} \gamma_{\mathrm{n}}}{\gamma_{\mathrm{s}} \tilde{J}} \tilde{n}-\gamma_{\mathrm{p}}\left(a_{\mathrm{r}}^{2}+a_{\mathrm{i}}^{2}-1\right)\right]\left(-b a_{\mathrm{r}}+a_{\mathrm{i}}\right)
\end{aligned}
$$




$$
\begin{aligned}
& -\xi_{\mathrm{i}} \gamma_{\mathrm{c}} \sin 2 \pi f_{\mathrm{i}} t \\
\frac{\mathrm{d} \tilde{n}}{\mathrm{~d} t} & -\left[\gamma_{\mathrm{s}}+\gamma_{\mathrm{n}}\left(a_{\mathrm{r}}^{2}+a_{\mathrm{i}}^{2}\right)\right] \tilde{n}-\gamma_{\mathrm{s}} \tilde{J}\left(a_{\mathrm{r}}^{2}+a_{\mathrm{i}}^{2}-1\right) \\
& +\frac{\gamma_{\mathrm{s}} \gamma_{\mathrm{p}}}{\gamma_{\mathrm{c}}} \tilde{J}\left(a_{\mathrm{r}}^{2}+a_{\mathrm{i}}^{2}\right)\left(a_{\mathrm{r}}^{2}+a_{\mathrm{i}}^{2}-1\right) \\
& +\gamma_{\mathrm{s}} m(1+\tilde{J}) \cos \left(2 \pi f_{\mathrm{m}} t+\varphi(t)\right)
\end{aligned}
$$

where $\gamma_{\mathrm{c}}=5.36 \times 10^{11} \mathrm{~s}^{-1}$ is the cavity decay rate, $\gamma_{\mathrm{s}}=$ $5.96 \times 10^{9} \mathrm{~s}^{-1}$ is the spontaneous carrier relaxation rate, $\gamma_{\mathrm{n}}=7.53 \times 10^{9} \mathrm{~s}^{-1}$ is the differential carrier relaxation rate, and $\gamma_{\mathrm{p}}=1.91 \times 10^{10} \mathrm{~s}^{-1}$ is the nonlinear carrier relaxation rate such that the relaxation resonance frequency $f_{\mathrm{r}}$ is $10.25 \mathrm{GHz}$ [26]. The normalized bias current above threshold is set at $\tilde{J}=1.222$. A linewidth enhancement factor, also known as the Henry factor of linewidth enhancement, $b=3.2$ quantifies the dependence of the index of refraction on the optical gain [48], [49]. The above parameters were extracted from a distributed-feedback laser [35]. The injection strength is defined as $\xi_{\mathrm{i}}=\eta\left|A_{\mathrm{i}}\right| /\left(\gamma_{\mathrm{c}}\left|A_{0}\right|\right)$, where $A_{0}$ is the free-running complex intracavity field amplitude, $A_{\mathrm{i}}$ is the injection field amplitude before entering the laser, and $\eta$ is the injection coupling rate [47], [49], [50]. The injection coupling rate is related to the structure of the laser, which was treated for different cases thoroughly in Ref. [51]. Once the alignment for injection into the laser is fixed, $\xi_{i}^{2}$ is proportional to the optical power of the injection light [49]. The last term in Eq. (3) is due to the uplink PSK current modulation, where the phase $\varphi(t)$ takes the value of 0 or $\pi$ when the data is 0 or 1 , respectively. In addition, a Langevin noise is used to simulate spontaneous emission noise of spontaneous emission rate $R_{\mathrm{s} p}=2.34 \times 10^{18} \mathrm{~V}^{2} \mathrm{~m}^{-2} \mathrm{~s}^{-1}$ [35]. Throughout the following simulation, second-order Runge-Kutta integration on Eqs. (1)-(3) is employed.

\section{RESUlts}

The numerical results are presented as follows. The generation of the P1 oscillation in the slave laser using optical injection without any uplink data is first introduced, where the tunability, double-locking quality, and locking range are addressed. Then, the results on the all-optical demodulation performance and the associated optimization of the injection condition are presented. Moreover, the dependencies of the BER on the input signal strength and bandwidth are examined, which exemplify the advantages of the P1 state over the stable locking state. Lastly, transmission issues induced by fiber dispersion are presented to compare the performances of alloptical and electrical demodulations.

\section{A. P1 Oscillation Generation}

Generation of the P1 state under only a continuous-wave optical injection from the master laser is considered. The intensity from the slave laser under the P1 state, unlike the stable locking state, oscillates due to undamping of the relaxation oscillation even without any current modulation. In the parameter space $\left(\xi_{\mathrm{i}}, f_{\mathrm{i}}\right)$, there exists a large region for $\mathrm{P} 1$ oscillation as long as $f_{\mathrm{i}}$ is larger than the requirement for a Hopf bifurcation from stable locking [26]. Figure 2

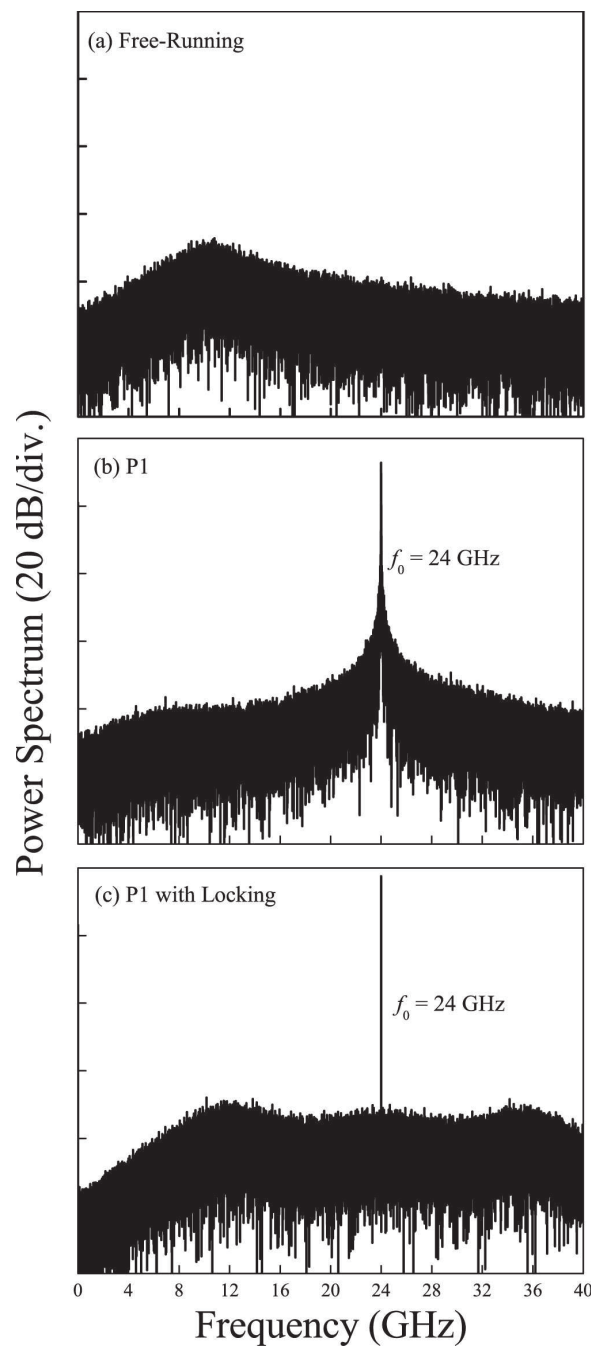

Fig. 2. Power spectra of the slave laser emission without uplink data. The laser is in the (a) free-running state with $\xi_{\mathrm{i}}=0$, (b) P1 state at $f_{0}=24 \mathrm{GHz}$ with $\left(\xi_{\mathrm{i}}, f_{\mathrm{i}}\right)=(0.18,12.7 \mathrm{GHz})$, and (c) P1 state locked by current modulation of $\left(m, f_{\mathrm{m}}\right)=(0.5,24 \mathrm{GHz})$.

shows the power spectra of the emission from the slave laser. In Fig. 2(a) the laser is in the free-running state, where the optical injection is off and no current modulation is applied. The power spectrum is a typical laser noise spectrum attributed to the spontaneous emission noise, where a broad peak at around the relaxation resonance frequency $f_{\mathrm{r}}=10.25 \mathrm{GHz}$ is observed. In Fig. 2(b), optical injection with $\left(\xi_{\mathrm{i}}, f_{\mathrm{i}}\right)=$ $(0.18,12.7 \mathrm{GHz})$ is applied, the slave laser is injection-locked into the P1 oscillation state. The power spectrum shows a clear peak at the P1 oscillation frequency $f_{0}=24 \mathrm{GHz}$, which is much larger than $f_{\mathrm{r}}$ due to the bandwidth enhancement effect of optical injection [26], [35]. However, the peak at $f_{0}$ is not very sharp because of the spontaneous emission noise. In Fig. 2(c), a current modulation of $\left(m, f_{\mathrm{m}}\right)=(0.5,24 \mathrm{GHz})$ is further applied to the slave laser. Because $f_{\mathrm{m}}=f_{0}$, the current modulation effectively locks the P1 oscillation. The power spectrum at $f_{0}$ is much sharpened. Thus, the laser is double-locked by both the optical injection and the current modulation. 


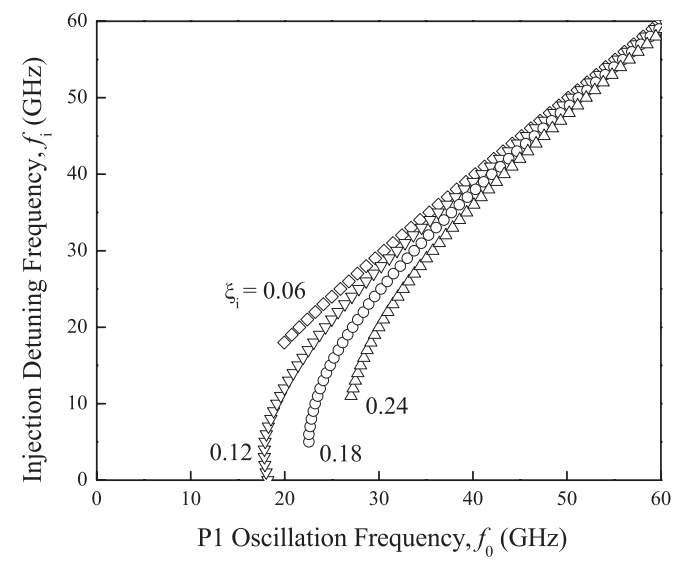

Fig. 3. Required injection detuning frequency $f_{\mathrm{i}}$ as a function of the $\mathrm{P} 1$ oscillation frequency $f_{0}$. No current modulation is applied. The injection strength is kept at $\xi_{\mathrm{i}}=0.06$ (diamonds), 0.12 (downward triangles), 0.18 (circles), and 0.24 (upward triangles).

1) Tunability: The $\mathrm{P} 1$ oscillation frequency $f_{0}$ can be widely tuned by controlling the injection parameters $\left(\xi_{\mathrm{i}}\right.$, $f_{\mathrm{i}}$ ) [47]. When no modulation is applied, Fig. 3 shows the required injection detuning frequency $f_{\mathrm{i}}$ as a function of the P1 oscillation frequency $f_{0}$. The injection strength is kept constant at $\xi_{\mathrm{i}}=0.06,0.12,0.18$, and 0.24 for the diamonds, down-triangles, circles, and up-triangles, respectively. The P1 oscillation frequency $f_{0}$ generally increases with $f_{\mathrm{i}}$ for each fixed $\xi_{\mathrm{i}}$. The lower ends for $f_{0}$ are the lower-bounds of P1 oscillation for each value of $\xi_{i}$.

Additionally, when a current modulation at $f_{\mathrm{m}}=f_{0}$ is applied to lock the P1 oscillation, the resultant modulation response is shown in Fig. 4. The modulation index is kept at $m=0.5$. The injection detuning frequency $f_{\mathrm{i}}$ is always tuned to vary $f_{0}$ before the current modulation is applied. For comparison, the modulation response without optical injection is shown by the squares in Fig. 4. The response peaks at around the relaxation resonance frequency $f_{\mathrm{r}}=10.25 \mathrm{GHz}$. The response drops drastically when the modulation frequency increases beyond $f_{\mathrm{r}}$. Clearly, Fig. 4 shows that optically injecting the slave laser into the P1 state enhances the modulation response. The enhancement increases with the injection strength $\xi_{\mathrm{i}}$. The P1 oscillation is generated by the optical injection alone without needing the current modulation, which is responsible for merely locking the oscillation.

2) Double Locking: The quality of double locking is monitored by the power spectrum of the slave laser emission, which can be quantified by the generated microwave power at the modulation frequency $f_{m}$ and the associated phase noise variance. Figure 5 shows such measurements as the input current modulation power to the slave laser is varied. The modulation frequency is set at $f_{\mathrm{m}}=24 \mathrm{GHz}$. Termination impedance of $50 \Omega$ is considered. The phase noise variance is estimated by integrating the averaged sidebands from $3 \mathrm{MHz}$ to $10 \mathrm{GHz}$ in the power spectrum normalized to the peak [52]. The circles in Fig. 5 show the results when optical injection is applied at $\left(\xi_{\mathrm{i}}, f_{\mathrm{i}}\right)=(0.18,12.7 \mathrm{GHz})$, which yields the P1 state at $f_{0}=f_{\mathrm{m}}$. The closed circles reveal insensitivity of the generated power to the input modulation power. This is

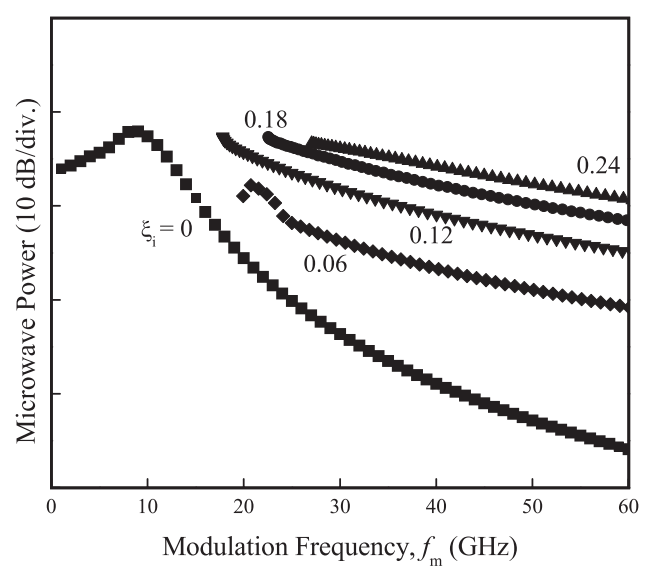

Fig. 4. Modulation response of the slave laser as a function of the modulation frequency $f_{\mathrm{m}}$. The optical injection strength is kept at $\xi_{\mathrm{i}}=0$ (squares), 0.06 (diamonds), 0.12 (downward triangles), 0.18 (circles), and 0.24 (upward triangles). Except for the case with $\xi_{\mathrm{i}}=0$, the injection detuning frequency $f_{\mathrm{i}}$ is always tuned to generate $\mathrm{P} 1$ oscillation at $f_{0}=f_{\mathrm{m}}$ before the current modulation is applied.

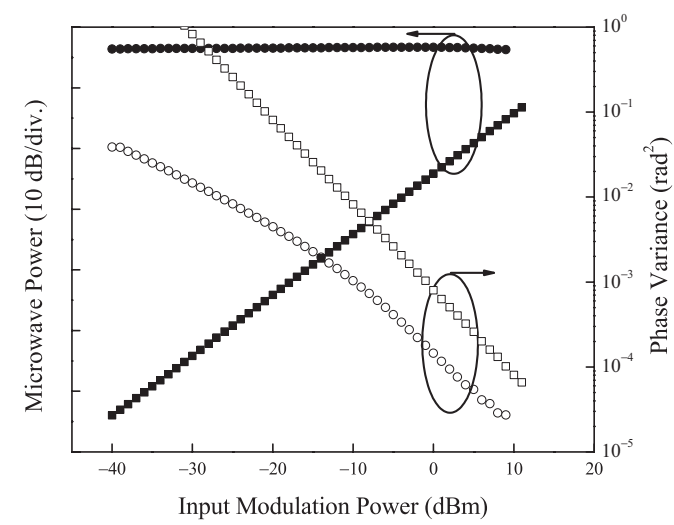

Fig. 5. Relative microwave power generated and the associated phase noise variance as functions of the input modulation power. The modulation frequency is set at $f_{\mathrm{m}}=24 \mathrm{GHz}$. For the circles, optical injection is applied at $\left(\xi_{\mathrm{i}}, f_{\mathrm{i}}\right)=(0.18,12.7 \mathrm{GHz})$ to obtain the $\mathrm{P} 1$ state at $f_{0}=f_{\mathrm{m}}$. For the squares, optical injection is turned off.

expected because the P1 oscillation is not caused by the current modulation. The open circles show monotonic reduction in phase noise with increasing input modulation power as the P1 oscillation is being progressively locked. By contrast, the squares in Fig. 5 are obtained when the optical injection is switched off. The closed squares confirm a linear dependency of the generated microwave power to the input modulation power. The phase variance is inversely proportional to the input power, as the open squares show. The phase noise originates from the relative intensity noise of the laser. It is verified in Fig. 5 that, as compared to the free-running laser, the doubly-locked laser gives stronger microwave power together with lower phase noise.

3) Locking Range: Double locking is possible even when $f_{\mathrm{m}}$ is tuned away from $f_{0}$ as long as $f_{\mathrm{m}}$ is sufficiently close to $f_{0}$. To quantify the locking characteristic of the P1 state, the statistical variance of the residual phase noise of the locked oscillation at $f_{\mathrm{m}}$ is investigated as the current modulation index $m$ varies. Figure 6 shows the results as a 


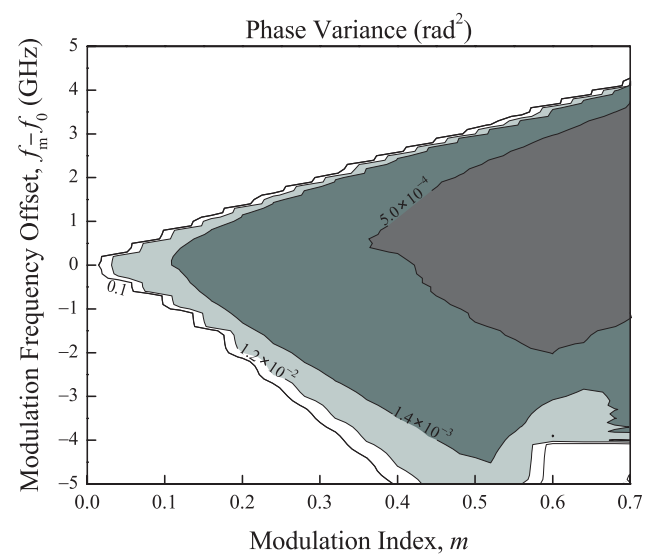

Fig. 6. Phase noise variance of the P1 state locked by a current modulation at frequency $f_{\mathrm{m}}$ and modulation index $m$. The slave laser is under injection of $\left(\xi_{\mathrm{i}}, f_{\mathrm{i}}\right)=(0.18,12.7 \mathrm{GHz})$ so that $f_{0}=24 \mathrm{GHz}$.

contour map in $\left(m, f_{\mathrm{m}}-f_{0}\right)$. The slave laser is under injection of $\left(\xi_{\mathrm{i}}, f_{\mathrm{i}}\right)=(0.18,12.7 \mathrm{GHz})$ to generate the $\mathrm{P} 1$ oscillation at $f_{0}=24 \mathrm{GHz}$ prior to applying the current modulation. The phase noise is contributed by the intrinsic spontaneous emission noise, the frequency difference $f_{\mathrm{m}}-f_{0}$, and, in some occasions, the emergence of other nonlinear dynamical states [26], [52]. For any fixed $m$, the phase variance is not necessarily the smallest at $f_{\mathrm{m}}=f_{0}$. Also, locking favors negative modulation frequency offset $f_{\mathrm{m}}-f_{0}$, which is not uncommon in the locking of lasers because of the antiguidance effect [53]. Define the locking range as the tolerable range of $f_{\mathrm{m}}$ that maintains a phase noise variance below $0.1 \mathrm{rad}^{2}$ [52]. It is seen in Fig. 6 that the locking range increases nearly linearly with the modulation index $m$. Subsequent results on the uplink transmission verify that the data bandwidth supported by the double-locked P1 state also increases nearly linearly with $m$, which is the modulation index on directly modulating the laser bias current. While the range of values for $m$ in consideration seems relatively large, it in not uncommon in the generation of high-frequency microwave signals to employ strong modulation on the bias current [34].

\section{B. Data Transmission}

Consider double locking of the slave laser using an uplink PSK signal. The data contained in the phase of the uplink microwave signal is transferred to the optical phase of the emission from the slave laser. Figure 7 shows the optical spectra from the slave laser under current modulation at $f_{\mathrm{m}}=$ $24 \mathrm{GHz}$ that carries 622-Mbps PSK uplink data. The current modulation index is kept at $m=0.5$. The frequency axis is offset to the free-running frequency of the slave laser. In Fig. 7(a), $\xi_{\mathrm{i}}=0$ as no optical injection is applied, the strongest peak appears at the free-running optical frequency at zero frequency offset. The sidebands at $\pm f_{\mathrm{m}}$ are due to the current modulation, but are very weak because $f_{\mathrm{m}}$ is much greater than the relaxation resonance frequency $f_{\mathrm{r}}$. In Fig. 7(b), the injection parameters are chosen as $\left(\xi_{\mathrm{i}}, f_{\mathrm{i}}\right)=(0.18,12.7 \mathrm{GHz})$ such that the $\mathrm{P} 1$ oscillation frequency is $f_{0}=24 \mathrm{GHz}$ prior to application of the current modulation. The arrow in Fig. 7(b)

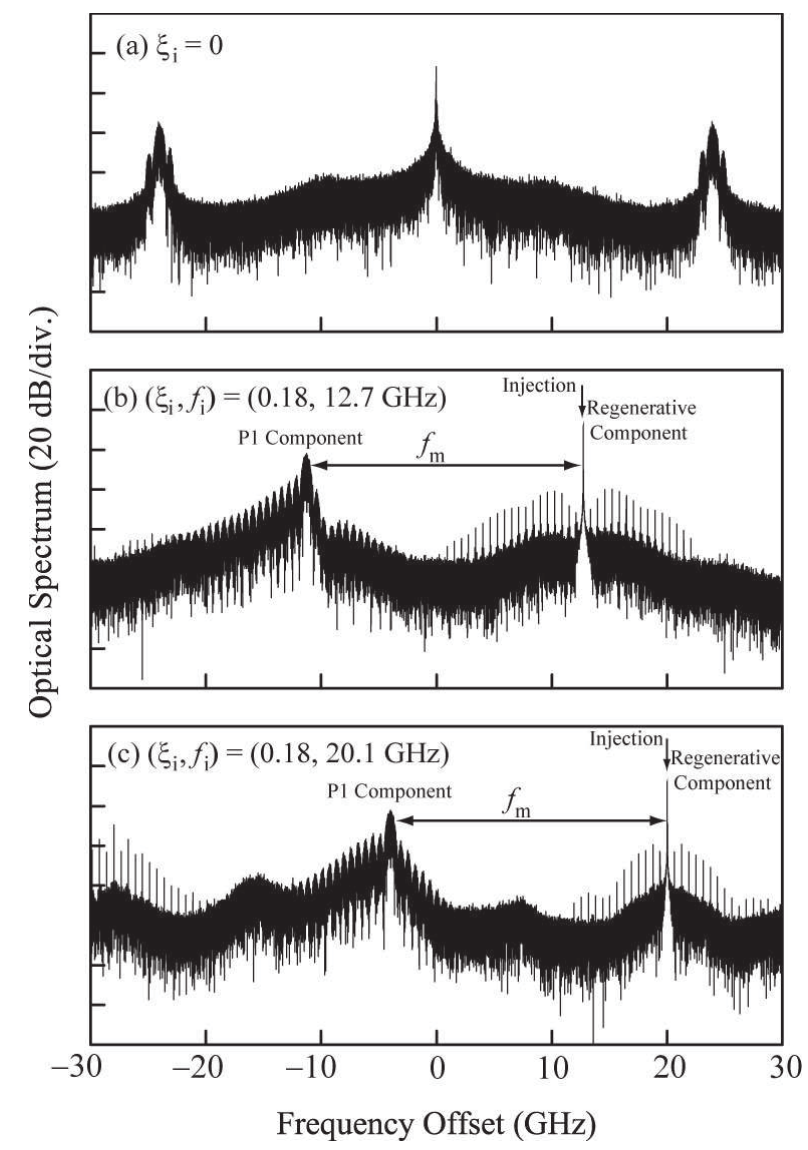

Fig. 7. Optical spectra from the slave laser modulated with an uplink PSK signal. The optical injection parameters are set at (a) $\xi_{\mathrm{i}}=0$, (b) $\left(\xi_{\mathrm{i}}, f_{\mathrm{i}}\right)=(0.18,12.7 \mathrm{GHz})$, and (c) $\left(\xi_{\mathrm{i}}, f_{\mathrm{i}}\right)=(0.18,20.1 \mathrm{GHz})$. The current modulation is of $\left(m, f_{\mathrm{m}}\right)=(0.5,24 \mathrm{GHz})$ with a data rate of $622 \mathrm{Mb} / \mathrm{s}$. The frequency axis is offset to the free-running frequency of the slave laser.

indicates the regenerative component from the master laser at frequency offset of $f_{\mathrm{i}}=12.7 \mathrm{GHz}$. The $\mathrm{P} 1$ oscillation of $f_{0}=24 \mathrm{GHz}$ generates the $\mathrm{P} 1$ component at the frequency offset of $f_{\mathrm{i}}-f_{0}=-11.3 \mathrm{GHz}$. The current modulation transfers the microwave PSK data at $f_{\mathrm{m}}=f_{0}=24 \mathrm{GHz}$ into optical PSK data on the P1 component, which are manifested as the sidebands around the P1 component in Fig. 7(b). Additionally, according to Fig. 6, tuning $f_{0}$ away from $f_{\mathrm{m}}$ can be considered to optimize the quality of double locking. This is achieved by varying the injection detuning frequency to $f_{\mathrm{i}}=20.1 \mathrm{GHz}$ and keeping the injection strength at $\xi_{\mathrm{i}}=0.18$ as shown in Fig. 7(c). Such injection generates P1 oscillation at $f_{0}=27.3 \mathrm{GHz}$ prior to the application of the uplink current modulation. The uplink signal carried at $f_{\mathrm{m}}=24 \mathrm{GHz}$ then locks the P1 oscillation. The uplink microwave PSK signal at $f_{\mathrm{m}}$ is still converted to an optical PSK signal carried by the locked $\mathrm{P} 1$ oscillation component at $f_{\mathrm{i}}-f_{\mathrm{m}}$. The central lobe of data at the P1 oscillation component becomes more symmetric, resulting in a better demodulation performance. Demodulation of the signal in Fig. 7(c) is investigated as follows.

1) Demodulation: The all-optical scheme in Fig. 1(a) is adopted for demodulation. The upstream signal in Fig. 7(c) is transmitted to the central office. It passes through the delayline interferometer and then the O/E converter in Fig. 1(a) to become the output. Figures 8(a), (b), and (c) show the time 


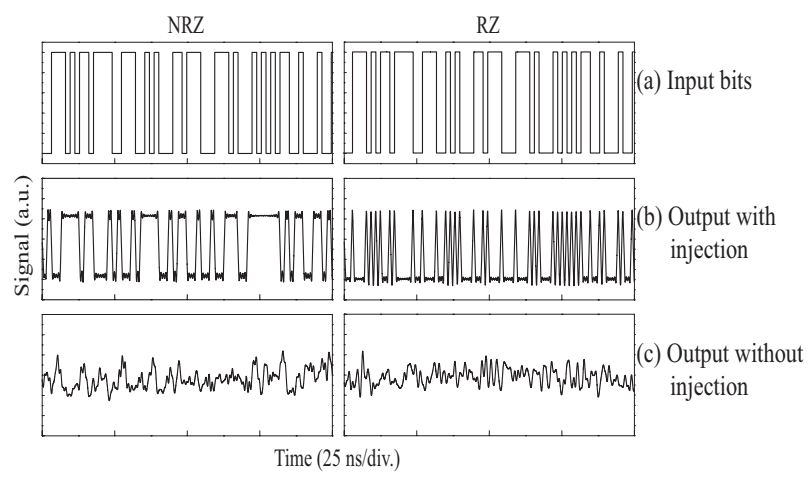

Fig. 8. Time series of (a) input bits, (b) output with optical injection, and (c) output without optical injection. One-bit (half-bit) delay is adopted in the optical differential demodulation that results in the NRZ (RZ) format in the left (right) column.

series of the uplink input bits, the output when the optical injection remains on, and the output when the optical injection is off, respectively. On the left column, the time-delay of the interferometer is set at one-bit duration so that consecutive bits are optically interfered. The output intensity of the interferometer is " 0 " and " 1 " when the optical phase difference of consecutive upstream bits is 0 and $\pi$, respectively. Thus, the upstream optical PSK signal in Fig. 7(c) is differentially demodulated to the output in Fig. 8(b), where the output bit is " 1 " if and only if there is a change in the input bits in Fig. 8(a). The output is in the non-return-to-zero (NRZ) format [39]. Estimation from the corresponding output eyediagram gives BER $=7.7 \times 10^{-13}$. For comparison, when the optical injection is switched off, the output in Fig. 8(c) does not give correct demodulation at all. On the right column, the time-delay of the interferometer is shortened to half-bit duration. The output is now in the return-to-zero (RZ) format as Fig. 8(b) shows, where each "1" corresponds to a change in the consecutive input bits Fig. 8(a). Again, when optical injection is switched off in Fig. 8(c), the data cannot be recovered successfully. Therefore, it is important to optically inject the laser into the P1 state in order to modulate the laser with the uplink signal. Data is transmitted upstream in optical PSK that enables all-optical demodulation, which does not require the high-speed $\mathrm{O} / \mathrm{E}$ converter and high-frequency local oscillator needed in the electrical scheme. Demodulation into NRZ is investigated in the rest of the paper.

2) Optimization: Based on the results in Fig. 6, best double locking requires tuning the P1 oscillation frequency $f_{0}$ slightly away from the the modulation frequency $f_{\mathrm{m}}$; therefore, minimization of the BER requires optimizing $f_{0}$ though varying the injection parameters $\left(\xi_{\mathrm{i}}, f_{\mathrm{i}}\right)$. The injection detuning frequency $f_{\mathrm{i}}$ can be conveniently tuned by adjusting the bias and temperature of the master laser. As a result, the BER is examined as a function of $f_{\mathrm{i}}$ in Fig. 9. A range of modulation index $m$ is considered. The modulation frequency is fixed at $f_{\mathrm{m}}=24 \mathrm{GHz}$. The injection strength is kept constant at $\xi_{\mathrm{i}}=0.18$. Figure 7 shows a minimum BER at $\left(f_{\mathrm{i}}, m\right)=(20.1 \mathrm{GHz}, 0.5)$ with details presented in Figs. 7(c) and 8 . The optimal $f_{\mathrm{i}}$ for minimum BER reduces slightly when $m$ decreases.

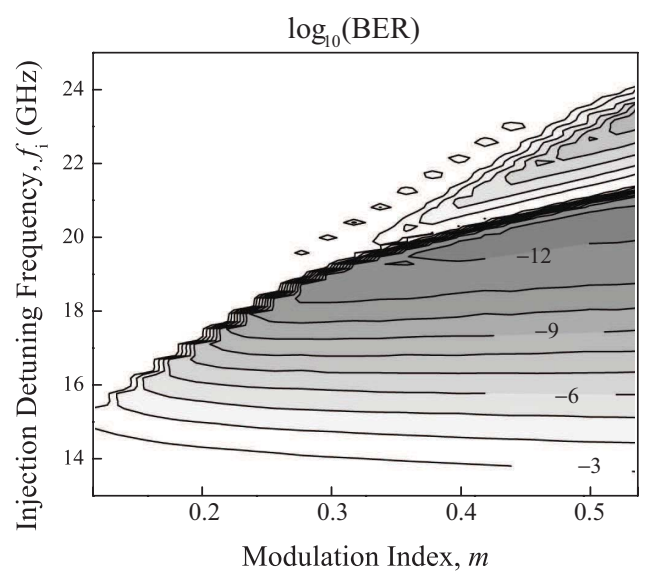

Fig. 9. BER as a function of the injection detuning frequency $f_{\mathrm{i}}$ and the modulation index $m$. Injection strength is kept constant at $\xi_{\mathrm{i}}=0.18$.

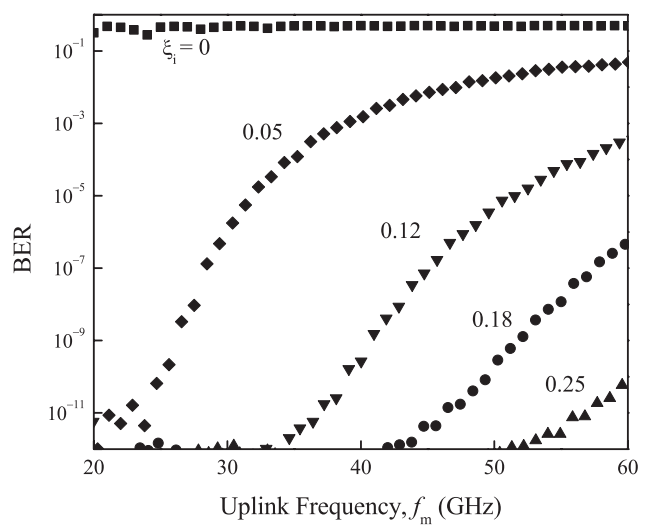

Fig. 10. BER versus $f_{\mathrm{m}}$ using different injection strengths as labeled. The data rate is kept constant at $622 \mathrm{Mb} / \mathrm{s}$. The injection detuning frequency $f_{\mathrm{i}}$ is always optimized to minimize the BER.

3) Residues: According to Fig. 7(c), the transfer of the uplink microwave PSK signal to the optical PSK signal on the P1 component through double locking is not perfect. First, the data sidebands around the P1 components are not exactly symmetric because residual intensity modulation exists. The intensity modulation can be demodulated by the O/E converter when the signal by-passes the interferometer in Fig. 1(a). However, it is found that the demodulated signal from intensity modulation is at least $35 \mathrm{~dB}$ weaker than that from the phase modulation. Second, Fig. 7(c) also reveals that both the regenerative component and the P1 oscillation component are modulated by data. To investigate the contributions from the two components individually, an optical filter is inserted immediately before the interferometer in Fig. 1(a). It is found that the recovered data is weakened by about $20 \mathrm{~dB}$ if the P1 oscillation component is filtered out, while the recovered data is basically unaffected if the regenerative component is filtered out. Therefore, data is mainly carried by PSK on the P1 component.

\section{Dependence on Uplink}

The performance of using the double-locked P1 state is characterized against the uplink signal properties. Figure 10 


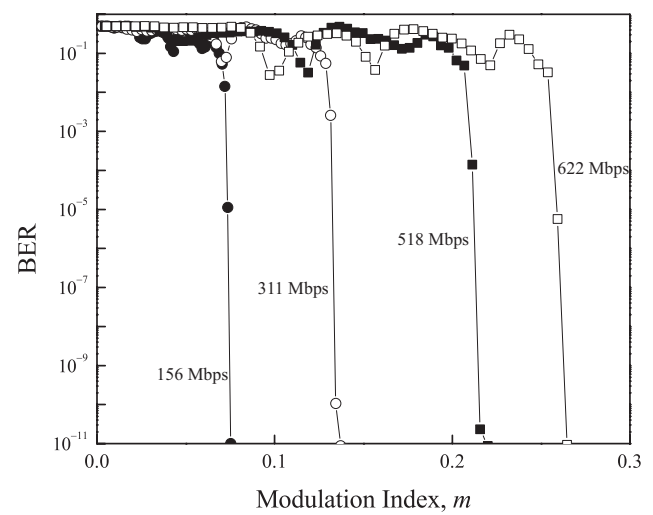

Fig. 11. BER versus $m$ at different data rates as labeled. The uplink frequency is $f_{\mathrm{m}}=24 \mathrm{GHz}$. The injection strength is kept constant at $\xi_{\mathrm{i}}=0.18$. The injection detuning frequency $f_{\mathrm{i}}$ is optimized for each data rate.

shows the BER as a function of the uplink subcarrier frequency $f_{\mathrm{m}}$. Different injection strengths are considered as labeled by $\xi_{\mathrm{i}}$, while the injection detuning frequency $f_{\mathrm{i}}$ is always optimized to minimize the BER. From Fig. 10, uplink transmission for $f_{\mathrm{m}}$ continuously tunable up to over $60 \mathrm{GHz}$ is possible at $\xi_{\mathrm{i}}=0.25$, where the BER remains below $10^{-9}$. Transmission is impossible for any $f_{\mathrm{m}}$ when the optical injection is off with $\xi_{\mathrm{i}}=0$ in Fig. 10 . In general, the BER improves as $\xi_{\mathrm{i}}$ increases or $f_{\mathrm{m}}$ reduces. Moreover, Fig. 11 shows the BER versus $m$ for different uplink data rates. The data rates are in accordance with the standard of Synchronous Optical Networking (SONET). The uplink modulation frequency is kept at $f_{\mathrm{m}}=24 \mathrm{GHz}$. The injection strength is kept constant at $\xi_{\mathrm{i}}=0.18$. For each data rate, the injection detuning frequency $f_{\mathrm{i}}$ is optimized to minimize the modulation index $m$ required for the smallest BER. It is observed that, as the data rate increases from $156 \mathrm{Mbps}$ to $622 \mathrm{Mbps}$, the required $m$ increases from 0.08 to 0.26 nearly linearly. Furthermore, the BER performances of uplink transmission using the P1 states, stable locking states, and free-running state are compared; as shown by the circles, triangles, and squares in Figs. 12 and 13. Three representative uplink frequencies of $f_{\mathrm{m}}=24 \mathrm{GHz}$ (dark symbols), $37 \mathrm{GHz}$ (gray symbols), and $60 \mathrm{GHz}$ (open symbols) are considered. For the P1 states, the injection parameters are set as $\left(\xi_{\mathrm{i}}, f_{\mathrm{i}}\right)=(0.18,20.1 \mathrm{GHz}),(0.35,31.5 \mathrm{GHz})$, and $(0.45$, $58.6 \mathrm{GHz}$ ) to minimize the BERs for $f_{\mathrm{m}}=24 \mathrm{GHz}, 37 \mathrm{GHz}$, and $60 \mathrm{GHz}$, respectively. For the stable locking states, the optical injection detuning frequency is tuned to $f_{\mathrm{i}}=0$. Such zero-detuning injection is often used for stable locking experiments [54]. For the free-running state, the injection is simply turned off by setting $\xi_{\mathrm{i}}=0$. The data rate is fixed at 622 Mbps in both Figs. 12 and 13.

1) Signal Power Range: Figure 12 shows the BER performances of the free-running state, stable locking states, and P1 states as functions of the modulation index $m$. Without optical injection, the laser cannot respond to uplink signals at $24 \mathrm{GHz}, 37 \mathrm{GHz}$, or $60 \mathrm{GHz}$ because the frequencies are much higher than the relaxation resonance frequency. The BER for the free-running state therefore remains very high as the squares show. With optical injection that stably locks the laser, the laser experiences enhancement in the

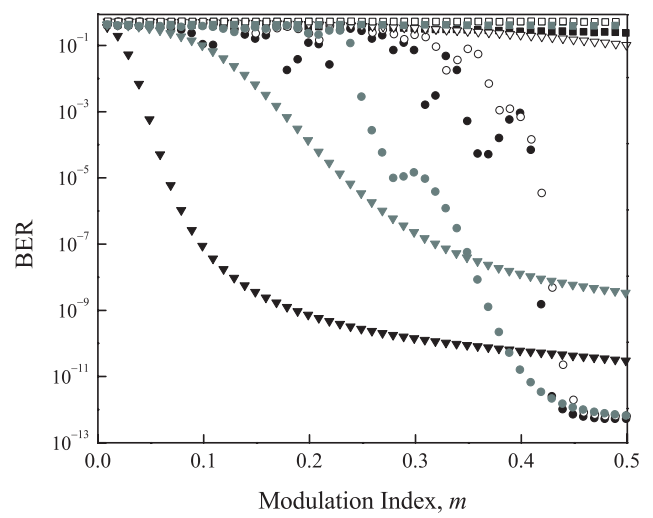

Fig. 12. BER versus $m$ for uplinks at $f_{\mathrm{m}}=24 \mathrm{GHz}$ (dark symbols), $37 \mathrm{GHz}$ (gray symbols), and $60 \mathrm{GHz}$ (open symbols). The slave laser is in the freerunning (squares), stable locking (triangles), and P1 states (circles).

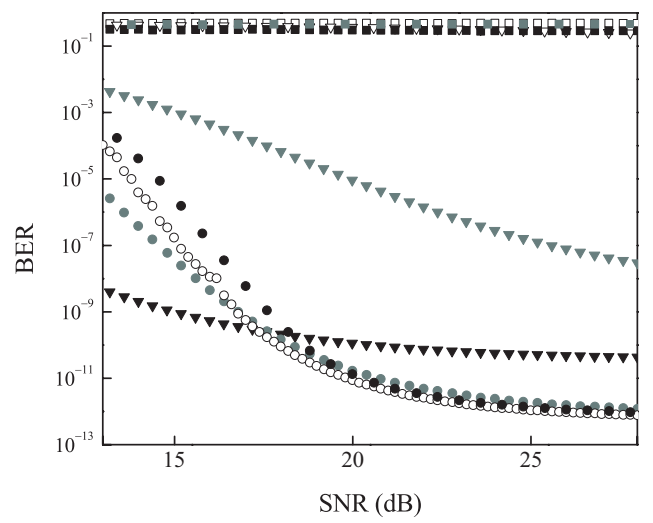

Fig. 13. BER versus SNR for uplinks at $f_{\mathrm{m}}=24 \mathrm{GHz}$ (dark symbols), $37 \mathrm{GHz}$ (gray symbols), and $60 \mathrm{GHz}$ (open symbols). The slave laser is in the free-running (squares), stable locking (triangles), and P1 states (circles).

modulation bandwidth. The BER reduces when $m$ increases as the triangles show; however, the reduction is gradual. The uplink at $60 \mathrm{GHz}$ resulted in a very high BER as shown by the open triangles. By contrast, when the optical injection drives the laser into the P1 states, the uplink data is modulated to the laser through double locking. The circles show drastic reduction of BER as $m$ increases beyond around 0.4. Transmission with BER below $10^{-9}$ is achieved even for the uplink at $60 \mathrm{GHz}$. Therefore, the P1 state outperforms the corresponding stable locking state when the uplink signal power, or equivalently, the modulation index is sufficiently strong.

2) Signal-to-Noise Ratio: The uplink electrical signal received by the base station may contain electrical noise due to electromagnetic interference and cross-talks. Such noise is different from the optical noise already considered in Section III. The electrical noise is modeled as an additive white Gaussian noise on the uplink signal. The BERs as functions of the uplink signal-to-noise ratio (SNR) are shown in Fig. 13. The modulation index is kept at $m=0.5$. For the free-running state, the squares show that no data can be recovered irrespective of the SNR. For the stable locking states, the triangles show gradual reduction of the BER as the SNR increases, but the reduction becomes insignificant as the 


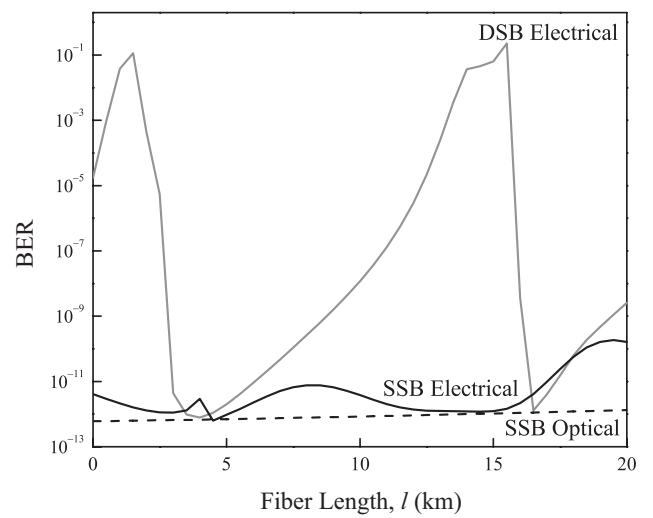

Fig. 14. BER versus $l$ for $f_{\mathrm{m}}=24 \mathrm{GHz}$. Demodulation is realized electrically on the DSB P1 state (gray curve), electrically on the SSB P1 state (dark curve), and all-optically on the SSB P1 state (dashed curve).

uplink frequency changes from $24 \mathrm{GHz}$ to $60 \mathrm{GHz}$. As for the P1 states, the circles show clear reduction of the BER as the SNR increases. There is also no obvious BER degradation when the uplink frequency $f_{\mathrm{m}}$ rises from $24 \mathrm{GHz}$ to $60 \mathrm{GHz}$ for the P1 states.

\section{Dispersion}

Although the upstream optical spectrum from the slave laser always consists of a central carrier together with sidebands separated by the subcarrier frequency $f_{\mathrm{m}}$, the sidebands are not necessarily of equal magnitude. Depending on the injection parameters, the spectrum can vary from being single-sided to double-sided. For instance, when $\left(\xi_{\mathrm{i}}, f_{\mathrm{i}}\right)=(0.18,20.1 \mathrm{GHz})$, the lower and upper optical sidebands differ by over $22.1 \mathrm{~dB}$, thus the laser is in a single sideband (SSB) P1 state. When instead $\left(\xi_{\mathrm{i}}, f_{\mathrm{i}}\right)=(0.01,23.6 \mathrm{GHz})$, the lower and upper sidebands are nearly equal in magnitude, thus the laser is in a double sideband (DSB) P1 state. Both P1 states are applicable to transmitting uplinks at $\left(m, f_{\mathrm{m}}\right)=(0.5,24 \mathrm{GHz})$. Suppose there is chromatic dispersion in the fiber. The DSB modulation would suffer from a microwave power penalty upon $\mathrm{O} / \mathrm{E}$ conversion, whereas the SSB modulation would not [26], [47], [55]. In general, the BER performances of the SSB and DSB P1 states are different. Figure 14 shows the BER as a function of the fiber length $l$ for both P1 states. The fiber dispersion is modeled by introducing a frequencydependent optical phase into to the upstream signal, where a group-velocity dispersion of $17 \mathrm{ps} / \mathrm{km}-\mathrm{nm}$ is considered as in a Corning SMF-28 fiber at $1.55 \mu \mathrm{m}$ [47]. Suppose the electrical demodulation scheme in Fig. 1(b) is employed. The microwave power after the $\mathrm{O} / \mathrm{E}$ converter is prone to the dispersioninduced power penalty. This causes the BER for the DSB P1 state to fluctuate significantly, as the gray curve in Fig. 14 shows. The dark curve shows that the BER fluctuation is less severe for the SSB P1 state, where the power penalty is very little. Nonetheless, when the all-optical demodulation scheme in Fig. 1(a) is employed, the problem of microwave power penalty becomes irrelevant. The corresponding dashed curve in Fig. 14 reveals that a low BER is maintained regardless of the fiber length.
It is worth mentioning that, in practice, fluctuations along the fiber can result in changes of the polarization of the injection light at the base station, which may affect the quality of optical injection locking. One solution is to employ a polarization maintaining fiber as the connection between the base station and the central office, thereby ensuring polarization alignment of the injection light and the slave laser. Alternatively, an active polarization compensator can be employed at the central office [56]. In the central office, by monitoring the state of polarization of the upstream light, a polarization controller for the downstream light can be varied to compensate for the fluctuations in the fiber and so optimizing the polarization alignment upon injection into the slave laser.

\section{CONCLUSion}

In conclusion, comprehensive numerical simulations are conducted to systematically characterize the performance of a recently proposed application of the double-locked semiconductor for uplink transmission. The laser is optically injected by a master laser into the P1 state, while the uplink signal further locks it to transfer the uplink data onto the upstream optical phase. The tuning range of the P1 frequency is shown to be much larger than the modulation bandwidth of the laser; thus the uplink subcarrier frequency can be much higher than the relaxation resonance frequency of the laser. Subcarrier frequencies reaching $60 \mathrm{GHz}$ can be supported by the laser with a relaxation resonance frequency of only $10.25 \mathrm{GHz}$. As compared to the stable injection locking state and the freerunning state, the P1 oscillation state is found to perform better in terms of the required uplink signal strength and SNR for low BER. The approach allows both electrical demodulation and all-optical demodulation, where the latter excels in immunity to fiber dispersion. The results illustrate the potential of using the P1 nonlinear dynamics in uplink communication.

\section{REFERENCES}

[1] R. Lang, "Injection locking properties of a semiconductor laser," IEEE J. Quantum Electron., vol. 18, no. 6, pp. 976-983, Jun. 1982.

[2] S. K. Hwang, H. F. Chen, and C. Y. Lin, "All-optical frequency conversion using nonlinear dynamics of semiconductor lasers," Opt. Lett., vol. 34, pp. 812-814, Mar. 2009.

[3] S. C. Chan, Q. Liu, Z. Wang, and K. S. Chiang, "Tunable negativetap photonic microwave filter based on a cladding-mode coupler and an optically injected laser of large detuning," Opt. Exp., vol. 19, pp. 12045-12052, Jun. 2011.

[4] S. C. Chan and J. M. Liu, "Tunable narrow-linewidth photonic microwave generation using semiconductor laser dynamics," IEEE $J$. Sel. Topics Quantum Electron., vol. 10, no. 5, pp. 1025-1032, Sep.Oct. 2004

[5] S. K. Hwang and D. H. Liang, "Effects of linewidth enhancement factor on period-one oscillations of optically injected semiconductor lasers," Appl. Phys. Lett., vol. 89, no. 6, pp. 061120-1-061120-3, Aug. 2006.

[6] V. Annovazzi-Lodi, G. Aromataris, M. Benedetti, and S. Merlo, "Secure chaotic transmission on a free-space optics data link," IEEE J. Quantum Electron., vol. 44, no. 11, pp. 1089-1095, Nov. 2008.

[7] Y. C. Wang, B. J. Wang, and A. B. Wang, "Chaotic correlation optical time domain reflectometer utilizing laser diode," IEEE Photon. Technol. Lett., vol. 20, no. 19, pp. 1636-1638, Oct. 2008.

[8] W. T. Wu, Y. H. Liao, and F. Y. Lin, "Noise suppressions in synchronized chaos lidars," Opt. Exp., vol. 18, no. 25, pp. 26155-26162, Dec. 2010. 
[9] A. Uchida, K. Amano, M. Inoue, K. Hirano, S. Naito, H. Someya, I. Oowada, T. Kurashige, M. Shiki, S. Yoshimori, K. Yoshimura, and P. Davis, "Fast physical random bit generation with chaotic semiconductor lasers," Nature Photon., vol. 2, pp. 728-732, Dec. 2008.

[10] V. Annovazzi-Lodi, S. Donati, and M. Manna, "Chaos and locking in a semiconductor laser due to external injection," IEEE J. Quantum Electron., vol. 30, no. 7, pp. 1537-1541, Jul. 1994.

[11] S. Wieczorek, T. B. Simpson, B. Krauskopf, and D. Lenstra, "Bifurcation transitions in an optically injected diode laser: Theory and experiment," Opt. Commun., vol. 215, nos. 1-3, pp. 125-134, Jan. 2003.

[12] S. Wieczorek, W. W. Chow, L. Chrostowski, and C. J. Chang-Hasnain, "Improved semiconductor-laser dynamics from induced population pulsation," IEEE J. Quantum Electron., vol. 42, no. 6, pp. 552-562, Jun. 2006.

[13] M. Pochet, N. A. Naderi, Y. Li, V. Kovanis, and L. F. Lester, "Tunable photonic oscillators using optically injected quantum-dash diode lasers," IEEE Photon. Technol. Lett., vol. 22, no. 11, pp. 763-765, Jun. 2010.

[14] Y. S. Juan and F. Y. Lin, "Photonic generation of broadly tunable microwave signals utilizing a dual-beam optically injected semiconductor laser," IEEE Photon. J., vol. 3, no. 4, pp. 644-650, Aug. 2011.

[15] F. Y. Lin, S. Y. Tu, C. C. Huang, and S. M. Chang, "Nonlinear dynamics of semiconductor lasers under repetitive optical pulse injection," IEEE J. Sel. Topics Quantum Electron., vol. 15, no. 3, pp. 604-611, May-Jun. 2009.

[16] S. C. Chan and W. K. S. Tang, "Chaotic dynamics of laser diodes with strongly modulated optical injection," Int. J. Bifurcat. Chaos, vol. 19, no. 10, pp. 3417-3424, 2009.

[17] S. L. Pan and J. P. Yao, "Wideband and frequency-tunable microwave generation using an optoelectronic oscillator incorporating a Fabry-Perot laser diode with external optical injection," Opt. Lett., vol. 35, no. 11, pp. 1911-1913, Jun. 2010.

[18] R. Vicente, C. R. Mirasso, and I. Fischer, "Simultaneous bidirectional message transmission in a chaos-based communication scheme," Opt. Lett., vol. 32, pp. 403-405, Feb. 2007.

[19] S. Tang and J. M. Liu, "Chaos synchronization in semiconductor lasers with optoelectronic feedback," IEEE J. Quantum Electron., vol. 39, no. 6, pp. 708-715, Jun. 2003.

[20] Y. H. Hong and K. A. Shore, "Influence of optical feedback timedelay on power-drops in vertical-cavity surface-emitting lasers," IEEE J. Quantum Electron., vol. 41, no. 8, pp. 1054-1057, Aug. 2005.

[21] M. T. Fathi and S. Donati, "Thickness measurement of transparent plates by a self-mixing interferometer," Opt. Lett., vol. 35, pp. 844-846, Jun. 2010.

[22] J. Liu, Z. M. Wu, and G. Q. Xia, "Dual-channel chaos synchronization and communication based on unidirectionally coupled VCSELs with polarization-rotated optical feedback and polarization-rotated optical injection," Opt. Exp., vol. 17, pp. 12619-12626, Jul. 2009.

[23] S. C. Chan, S. K. Hwang, and J. M. Liu, "Radio-over-fiber AM-to-FM upconversion using an optically injected semiconductor laser," Opt. Lett., vol. 31, pp. 2254-2256, Aug. 2006.

[24] L. Chrostowski, X. X. Zhao, and C. J. Chang-Hasnian, "Microwave performance of optically injection-locked VCSELs," IEEE Trans. Microw. Theory Tech., vol. 54, no. 2, pp. 788-796, Feb. 2006.

[25] E. K. Lau, H. K. Sung, and M. C. Wu, "Frequency response enhancement of optical injection-locked laser," IEEE J. Quantum Electron., vol. 44, no. 1, pp. 90-99, Jan. 2008.

[26] S. C. Chan, "Analysis of an optically injected semiconductor laser for microwave generation," IEEE J. Quantum Electron., vol. 46, no. 3, pp. 421-428, Mar. 2010.

[27] X. Q. Qi, J. M. Liu, X. P. Zhang, and L. Xie, "Fiber dispersion and nonlinearity influences on transmissions of AM and FM data modulation signals in radio-over-fiber system," IEEE J. Quantum Electron., vol. 46, no. 8, pp. 1170-1177, Aug. 2010.

[28] X. Q. Qi and J. M. Liu, "Photonic microwave applications of the dynamics of semiconductor lasers," IEEE J. Sel. Topics Quantum Electron., vol. 17, no. 5, pp. 1198-1211, Sep.-Oct. 2011.

[29] A. J. Seeds and K. J. Williams, "Microwave photonics," J. Lightw. Technol., vol. 24, no. 3, pp. 4628-4641, Dec. 2006.

[30] J. Capmany and D. Novak, "Microwave photonics combines two worlds," Nature Photon., vol. 1, pp. 319-330, Jun. 2007.

[31] Z. S. Jia, J. J. Yu, G. Ellinas, and G. K. Chang, "Key enabling technologies for optical-wireless networks: Optical millimeter-wave generation, wavelength reuse, and architecture," J. Lightw. Technol., vol. 25, no. 11, pp. 3452-3471, Nov. 2007.

[32] J. P. Yao, "Microwave photonics," J. Lightw. Technol., vol. 27, no. 3, pp. 314-335, Feb. 2009.
[33] X. Fu, C. Cui, and S. C. Chan, "Optically injected semiconductor laser for photonic microwave frequency mixing in radio-over-fiber," $J$. Electromagn. Waves Appl., vol. 24, no. 7, pp. 849-860, 2010.

[34] H. Shams, P. Perry, P. M. Anandarajah, and L. P. Barry, "Modulated millimeter-wave generation by external injection of a gain switched laser," IEEE Photon. Technol. Lett., vol. 23, no. 7, pp. 447-449, Apr. 2011.

[35] S. K. Hwang, J. M. Liu, and J. K. White, "35-GHz intrinsic bandwidth for direct modulation in $1.3-\mu \mathrm{m}$ semiconductor lasers subject to strong injection locking," IEEE Photon. Technol. Lett., vol. 16, no. 4, pp. 972974, Apr. 2004.

[36] H. K. Sung, E. K. Lau, and M. C. Wu, "Optical single sideband modulation using strong optical injection-locked semiconductor lasers," IEEE Photon. Technol. Lett., vol. 19, no. 13, pp. 1005-1007, Jul. 2007.

[37] A. Ng'oma, D. Fortusini, D. Parekh, W. J. Yang, M. Sauer, S. Benjamin, W. Hofmann, M. C. Amann, and C. J. Chang-Hasnain, "Performance of a multi-Gb/s $60 \mathrm{GHz}$ radio over fiber system employing a directly modulated optically injection-locked VCSEL," J. Lightw. Technol., vol. 28 , no. 16, pp. 2436-2444, Aug. 2010.

[38] T. B. Simpson, J. M. Liu, and A. Gavrielides, "Bandwidth enhancement and broadband noise reduction in injection-locked semiconductor lasers," IEEE Photon. Technol. Lett., vol. 7, no. 7, pp. 709-711, Jul. 1995.

[39] C. Cui, X. Fu, and S. C. Chan, "Double-locked semiconductor laser for radio-over-fiber uplink transmission," Opt. Lett., vol. 34, no. 24, pp. 3821-3823, Dec. 2009.

[40] T. B. Simpson and F. Doft, "Double-locked laser diode for microwave photonics applications," IEEE Photon. Technol. Lett., vol. 11, no. 11, pp. 1476-1478, Nov. 1999.

[41] C. Lim, A. Nirmalathas, and D. Novak, "Techniques for multichannel data transmission using a multisection laser in millimeter-wave fiberradio systems," IEEE Trans. Microw. Theory Tech., vol. 47, no. 7, pp. 1351-1357, Jul. 1999.

[42] A. Kaszubowska, L. Hu, and L. P. Barry, "Remote downconversion with wavelength reuse for the radio/fiber uplink connection," IEEE Photon. Technol. Lett., vol. 18, no. 4, pp. 562-564, Feb. 2006.

[43] Y. Q. Song, X. P. Zheng, H. Y. Zhang, Y. L. Guo, and B. K. Zhou, "All-optical subcarrier demodulation in upstream link of millimeter-wave radio over fiber system," Opt. Lett., vol. 32, no. 15, pp. 2248-2250, Aug. 2007.

[44] C. Cui, X. Fu, and S. C. Chan, "Optically controlled radio-over-fiber uplink using double-locked semiconductor lasers," in Proc. OptoElectron. Commun. Conf., Jul. 2010, pp. 718-719.

[45] S. Wieczorek, T. B. Simpson, B. Krauskopf, and D. Lenstra, "Global quantitative predictions of complex laser dynamics," Phys. Rev. E, vol. 65, no. 4, pp. 045207R-1-045207R-4, Apr. 2002.

[46] T. B. Simpson, J. M. Liu, and A. Gavrielides, "Small-signal analysis of modulation characteristics in a semiconductor laser subject to strong optical injection," IEEE J. Quantum Electron., vol. 32, no. 8, pp. 14561468, Aug. 1996.

[47] S. C. Chan, S. K. Hwang, and J. M. Liu, "Period-one oscillation for photonic microwave transmission using an optically injected semiconductor laser," Opt. Exp., vol. 15, no. 22, pp. 14921-14935, Oct. 2007.

[48] C. H. Henry, "Phase noise in semiconductor lasers," J. Lightw. Technol., vol. 4, no. 3, pp. 298-311, Mar. 1986.

[49] T. B. Simpson, J. M. Liu, K. F. Huang, and K. Tai, "Nonlinear dynamics induced by external optical injection in semiconductor lasers," Quantum Semiclass. Opt., vol. 9, pp. 765-784, Oct. 1997.

[50] T. B. Simpson, J. M. Liu, A. Gavrielides, V. Kovanis, and P. M. Alsing, "Period-doubling route to chaos in a semiconductor laser subject to optical injection," Appl. Phys. Lett., vol. 64, no. 26, pp. 3539-3541, Jun. 1994.

[51] F. Kefelian and P. Gallion, "Locking and noise properties of multisection semiconductor lasers with optical injection. Application to Fabry-Pérot and DFB cavities," IEEE J. Quantum Electron., vol. 44, no. 6, pp. 547560, Jun. 2008.

[52] S. C. Chan and J. M. Liu, "Microwave frequency division and multiplication using an optically injected semiconductor laser," IEEE J. Quantum Electron., vol. 41, no. 9, pp. 1142-1147, Sep. 2005.

[53] S. K. Hwang, J. M. Liu, and J. K. White, "Characteristics of period-one oscillations in semiconductor lasers subject to optical injection," IEEE J. Sel. Topics Quantum Electron., vol. 10, no. 5, pp. 974-981, Sep.-Oct. 2004.

[54] M. Pochet, N. A. Naderi, N. Terry, V. Kovanis, and L. F. Lester, "Dynamic behavior of an injection-locked quantum-dash Fabry-Perot laser at zero-detuning," Opt. Exp., vol. 17, pp. 20623-20630, Nov. 2009. 
[55] C. Lim, D. Novak, A. Nirmalathas, and G. H. Smith, "Dispersioninduced power penalties in millimeter-wave signal transmission using multisection DBR semiconductor laser," IEEE Trans. Microw. Theory Tech., vol. 49, no. 2, pp. 288-296, Feb. 2001.

[56] H. Sunnerud, C. Xie, M. Karlsson, R. Samuelsson, and P. A. Andrekson, "A comparison between different PMD compensation techniques," $J$. Lightw. Technol., vol. 20, no. 3, pp. 368-378, Mar. 2002.

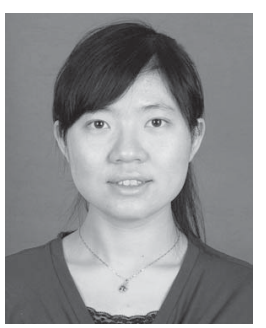

Cuicui Cui received the B.Eng. degree in electronic science and technology from the Harbin Institute of Technology, Harbin, China, and the M.Phil. degree in electronic engineering with the City University of Hong Kong, Hong Kong, in 2008 and 2010, respectively.

Her current research interests include microwave photonics and laser nonlinear dynamics.

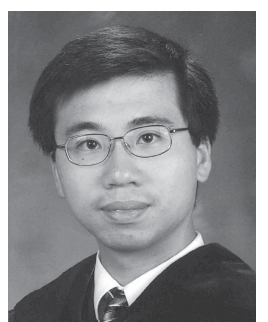

Sze-Chun Chan (S'98-M'07) received the B.Eng. degree in electrical and electronic engineering from the University of Hong Kong, Hong Kong, and the M.S. and Ph.D. degrees in electrical engineering from the University of California, Los Angeles, in 2001, 2004, and 2007, respectively.

$\mathrm{He}$ is currently an Assistant Professor of Electronic Engineering with the City University of Hong Kong, Hong Kong. His current research interests include laser nonlinear dynamics, microwave photonics, radio-over-fiber technology, optical chaos generation, and biological applications of semiconductor lasers. Dr. Chan received the Dr. Bor-Uei Chen Scholarship of the Photonics Society of Chinese-Americans in 2007. 